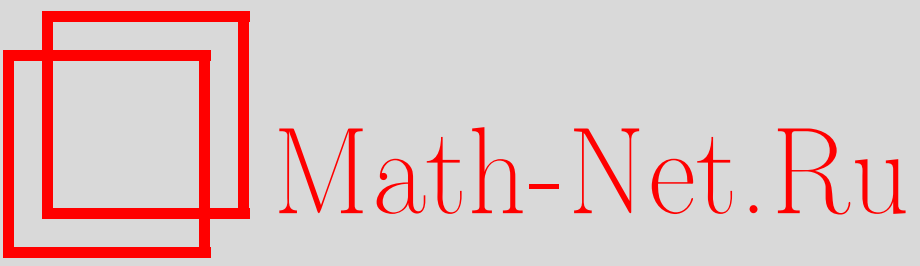

В. В. Жаринов, Соответствия Беклунда для эволюционных уравнений в многомерном пространстве, ТМФ, 2006, том 147, номер 1, 3-13

DOI: https://doi.org/10.4213/tmf2019

Использование Общероссийского математического портала Math-Net.Ru подразумевает, что вы прочитали и согласны с пользовательским соглашением

http://www . mathnet.ru/rus/agreement

Параметры загрузки:

IP : 54.147 .182 .235

26 апреля 2023 г., 16:05:18 
ТЕОРЕТИЧЕСКАЯ

И МАТЕМАТИЧЕСКАЯ

ФИЗИКА

Том 147, № 1

апрель, 2006

(C) 2006 г.

В. В. Жаринов*

\section{СООТВЕТСТВИЯ БЕКЛУНДА ДЛЯ ЭВОЛЮЦИОННЫХ УРАВНЕНИЙ В МНОГОМЕРНОМ ПРОСТРАНСТВЕ}

Цель предлагаемой статьи - развитие техники вычислений, позволяющей осуществлять эффективный алгебро-геометрический (групповой) анализ уравнений в частных производных с произвольным числом независимых переменных. Дано полное описание важного класса уравнений многомерной эволюции, допускающих соответствия Беклунда заданного вида. В частности, оказалось, что изученный класс достаточно широк, хотя в одномерном случае он богаче благодаря отсутствию условия равенства смешанных производных.

Ключевые слова: соответствие Беклунда, эволюционные системы, дифференциальные связи.

\section{1. ОБОЗНАЧЕНИЯ И ОПРЕДЕЛЕНИЯ}

Будем использовать следующие мультииндексные обозначения: $t$ - независимая переменная (время); $x=\left(x^{\alpha}\right)$ - независимые переменные (пространство), $1 \leqslant \alpha \leqslant D$; $u, v$ - зависимые переменные; $\mathbf{u}=\left(u, u_{x}, u_{x x}, \ldots\right), \mathbf{v}=\left(v, v_{x}, v_{x x}, \ldots\right)$ - дифференциальные переменные, где $u_{x}=\left(u_{\alpha}\right), \quad v_{x}=\left(v_{\alpha}\right), \quad u_{x x}=\left(u_{\alpha \beta}=u_{\beta \alpha}\right)$, $v_{x x}=\left(v_{\alpha \beta}=u_{\beta \alpha}\right), \ldots, 1 \leqslant \alpha, \beta, \ldots \leqslant D$.

Соответствие Беклунда для двух эволюционных уравнений

$$
u_{t}=f(t, x, \mathbf{u}), \quad v_{t}=g(t, x, \mathbf{v})
$$

есть дифференциальная связь

$$
R_{\alpha}(t, x, \mathbf{u}, \mathbf{v})=0, \quad 1 \leqslant \alpha \leqslant D
$$

обладающая следующим характеризующим свойством: всякое решение $u=$ $U(t, x)$ уравнения $u_{t}=f$ после подстановки в уравнения связи $R_{\alpha}=0$ приводит к максимально переопределенной системе уравнений

$$
\left.R_{\alpha}(t, x, \mathbf{u}, \mathbf{v})\right|_{\mathbf{u}=\mathbf{U}(t, x)}=0, \quad 1 \leqslant \alpha \leqslant D,
$$

*Математический институт им. В. А. Стеклова РАН, Москва, Россия. E-mail: zharinov@mi.ras.ru 
для нахождения семейства решений $v=V(t, x)$ уравнения $v_{t}=g$, и аналогичным свойством, позволяющим по данному решению уравнения $v_{t}=g$ строить семейство решений уравнения $u_{t}=f$. Здесь и ниже

$$
U_{\alpha}(t, x)=\frac{\partial U(t, x)}{\partial x^{\alpha}}, \quad U_{\alpha \beta}(t, x)=\frac{\partial^{2} U(t, x)}{\partial x^{\alpha} \partial x^{\beta}}, \quad \ldots, \quad 1 \leqslant \alpha, \beta, \ldots \leqslant D .
$$

Заметим, что такая конструкция не является преобразованием в обычном смысле в силу своей взаимной неоднозначности, так что естественно использовать более обтекаемый термин соответствие. В общем виде соответствия Беклунда для систем уравнений в частных производных описаны в работах [1], [2] (см. также [3]). В частности, система $R_{\alpha}=0,1 \leqslant \alpha \leqslant D$, будет обладать требуемыми свойствами тогда и только тогда, когда выполнено условие согласования

$$
\left.\overline{\partial_{t} R_{\alpha}} \equiv\left[\partial_{t} R_{\alpha}\right]\right|_{\mathcal{R}}=0, \quad 1 \leqslant \alpha \leqslant D
$$

где

$$
\begin{aligned}
\partial_{\alpha} & =\frac{\partial}{\partial x^{\alpha}}+\left(u_{\alpha} \frac{\partial}{\partial u}+v_{\alpha} \frac{\partial}{\partial v}\right)+\sum_{\beta}\left(u_{\alpha \beta} \frac{\partial}{\partial u_{\beta}}+v_{\alpha \beta} \frac{\partial}{\partial v_{\beta}}\right)+\cdots, \quad 1 \leqslant \alpha \leqslant D ; \\
\partial_{t} & =\frac{\partial}{\partial t}+\left(f \frac{\partial}{\partial u}+g \frac{\partial}{\partial v}\right)+\sum_{\beta}\left(\left(\partial_{\beta} f\right) \frac{\partial}{\partial u_{\beta}}+\left(\partial_{\beta} g\right) \frac{\partial}{\partial v_{\beta}}\right)+\cdots ; \\
\mathcal{R} & =\left\{R_{\alpha}=\partial_{\beta} R_{\alpha}=\partial_{\gamma} \partial_{\beta} R_{\alpha}=\cdots=0,1 \leqslant \alpha, \beta, \gamma, \cdots \leqslant D\right\}
\end{aligned}
$$

запись $\bar{F}$ или $\left.F\right|_{\mathcal{R}}$ означает сужение функции $F$ на поверхность $\mathcal{R}$ в пространстве переменных $t, x, \mathbf{u}, \mathbf{v}$.

Полные дифференцирования $\partial_{\alpha}$ характеризуются свойством

$$
\left.\left[\partial_{\alpha} F(t, x, \mathbf{u}, \mathbf{v})\right]\right|_{\mathbf{u}=\mathbf{U}(t, x), \mathbf{v}=\mathbf{V}(t, x)}=\frac{\partial}{\partial x^{\alpha}}\left[\left.F(t, x, \mathbf{u}, \mathbf{v})\right|_{\mathbf{u}=\mathbf{U}(t, x), \mathbf{v}=\mathbf{V}(t, x)}\right]
$$

для всех функций $F(t, x, \mathbf{u}, \mathbf{v}), U(t, x), V(t, x)$ и всех $1 \leqslant \alpha \leqslant D$. В свою очередь, эволюционное дифференцирование $\partial_{t}$ характеризуется следующим свойством: для данных функций $U(t, x), \quad V(t, x)$ равенство

$$
\left.\left[\partial_{t} F(t, x, \mathbf{u}, \mathbf{v})\right]\right|_{\mathbf{u}=\mathbf{U}(t, x), \mathbf{v}=\mathbf{V}(t, x)}=\frac{\partial}{\partial t}\left[\left.F(t, x, \mathbf{u}, \mathbf{v})\right|_{\mathbf{u}=\mathbf{U}(t, x), \mathbf{v}=\mathbf{V}(t, x)}\right]
$$

справедливо для всех функций $F(t, x, \mathbf{u}, \mathbf{v})$ тогда и только тогда, когда $U(t, x)$ и $V(t, x)$ суть решения уравнений $u_{t}=f$ и $v_{t}=g$, соответственно. Очевидно, что коммутаторы $\left[\partial_{\alpha}, \partial_{\beta}\right]=0,\left[\partial_{\alpha}, \partial_{t}\right]=0,1 \leqslant \alpha, \beta \leqslant D$. Для произвольных функций $R_{\alpha}(t, x, \mathbf{u}, \mathbf{v}), \quad 1 \leqslant \alpha \leqslant D$, поверхность $\mathcal{R}$ характеризуется следующим свойством: полные дифференцирования $\partial_{\alpha}$ допускают сужения $\bar{\partial}_{\alpha}$ с пространства переменных $t, x, \mathbf{u}, \mathbf{v}$ на $\mathcal{R}$, так что

$$
\overline{\partial_{\alpha} F}=\bar{\partial}_{\alpha} \bar{F}, \quad 1 \leqslant \alpha \leqslant D,
$$

для всех $F(t, x, \mathbf{u}, \mathbf{v})$ (напомним, что черта сверху означает сужение на поверхность $\mathcal{R})$. 
В предлагаемой работе рассматривается случай

$$
\begin{aligned}
u_{t} & =f=\sum_{\alpha, \beta} a^{\alpha \beta}\left(u, u_{x}\right) u_{\alpha \beta}+\phi\left(u, u_{x}\right), \\
v_{t} & =g=\sum_{\alpha, \beta} b^{\alpha \beta}\left(v, v_{x}\right) v_{\alpha \beta}+\psi\left(v, v_{x}\right), \\
R_{\alpha} & =R_{\alpha}\left(u, u_{x}, v, v_{x}\right)=0, \quad 1 \leqslant \alpha \leqslant D,
\end{aligned}
$$

причем налагаются естественные условия невырожденности, которые будут конкретизированы ниже. Учитывая, что почти во всех известных примерах соответствий Беклунда размерность пространства $D=1$, основное внимание уделяется случаю $D \geqslant 2$. Работа выполнена в рамках алгебро-геометрического подхода к уравнениям в частных производных (см., например, [2], [4]). В частности, по умолчанию все функции предполагаются достаточно гладкими, т.е. имеющими непрерывные частные производные необходимых порядков, а все рассуждения носят локальный характер, т.е. справедливы в какой-либо окрестности фиксированной точки общего положения (примерно так, как это делается в теореме о неявной функции).

\section{2. ДОПУСТИМЫЕ ДИФФЕРЕНЦИАЛЬНЫЕ СВЯЗИ}

Пусть $D \geqslant 2$ и выполнены условия невырожденности:

$$
\operatorname{det}\left\|\frac{\partial R_{\alpha}}{\partial u_{\beta}}\right\| \neq 0, \quad \operatorname{det}\left\|\frac{\partial R_{\alpha}}{\partial v_{\beta}}\right\| \neq 0 .
$$

В частности, система (1.4) разрешима относительно переменных $v_{\alpha}$,

$$
v_{\alpha}=r_{\alpha}\left(u, u_{x}, v\right), \quad 1 \leqslant \alpha \leqslant D, \quad \operatorname{det}\left\|\frac{\partial r_{\alpha}}{\partial u_{\beta}}\right\| \neq 0 .
$$

Явный вид функций $r_{\alpha}$ определяется условием равенства смешанных производных:

$$
v_{\alpha \beta}=\partial_{\alpha} v_{\beta}=\partial_{\beta} v_{\alpha}=v_{\beta \alpha},
$$

т.e.

$$
\overline{\partial_{\alpha} r_{\beta}}=\overline{\partial_{\beta} r_{\alpha}}, \quad 1 \leqslant \alpha, \beta \leqslant D,
$$

или, подробнее,

$$
r_{\beta u} u_{\alpha}+\sum_{\gamma} r_{\beta u_{\gamma}} u_{\alpha \gamma}+r_{\beta v} r_{\alpha}=r_{\alpha u} u_{\beta}+\sum_{\gamma} r_{\alpha u_{\gamma}} u_{\beta \gamma}+r_{\alpha v} r_{\beta},
$$

где индексы $u, v, u_{\alpha}, \ldots$ означают соответствующие частные производные, например, $r_{\beta u}=\partial r_{\beta} / \partial u$. Несложный, хотя и кропотливый анализ показывает, что равенство $(2.3)$ имеет место тогда и только тогда, когда существуют функция $R(u, v), R_{u} \neq 0$, $R_{v} \neq 0$, и постоянные $c_{\alpha}$ такие, что

$$
r_{\alpha}=-\frac{R_{u} u_{\alpha}+c_{\alpha}}{R_{v}}, \quad 1 \leqslant \alpha \leqslant D .
$$

В симметричной форме допустимая дифференциальная связь имеет вид

$$
R_{\alpha}=\partial_{\alpha} R(u, v)+c_{\alpha}=0, \quad 1 \leqslant \alpha \leqslant D .
$$




\section{3. ПЕРВЫЕ СЛЕДСТВИЯ ИЗ УСЛОВИЯ СОГЛАСОВАНИЯ}

Подставляя выражение (2.4) в условие согласования (1.1), получим

$$
\begin{aligned}
\overline{\partial_{t} R_{\alpha}}=\overline{\partial_{t}\left(\partial_{\alpha} R(u, v)+c_{\alpha}\right)}=\overline{\partial_{t} \partial_{\alpha} R}=\bar{\partial}_{\alpha}\left(\overline{\partial_{t} R}\right)= & \\
& =\bar{\partial}_{\alpha}\left(R_{u} \overline{u_{t}}+R_{v} \overline{v_{t}}\right)=\bar{\partial}_{\alpha}\left(R_{u} \bar{f}+R_{v} \bar{g}\right)=0, \quad 1 \leqslant \alpha \leqslant D .
\end{aligned}
$$

В нашем случае согласно (1.2) и (1.3)

$$
\begin{aligned}
& \bar{f}=\sum_{\beta, \gamma} \overline{a^{\beta \gamma}} \overline{u_{\beta \gamma}}+\bar{\phi}=\sum_{\beta, \gamma} \overline{a^{\beta \gamma}} \overline{\overline{u_{\beta \gamma}}}+\cdots, \\
& \bar{g}=\sum_{\beta, \gamma} \overline{b^{\beta \gamma}} \overline{v_{\beta \gamma}}+\bar{\psi}=\sum_{\beta, \gamma} \overline{b^{\beta \gamma}} \overline{v_{\beta \gamma}}+\cdots,
\end{aligned}
$$

где многоточия здесь и далее обозначают члены, содержащие дифференциальные переменные низших порядков. Следовательно,

$$
\bar{\partial}_{\alpha} \sum_{\beta, \gamma}\left(R_{u} \overline{a^{\beta \gamma}} \overline{u_{\beta \gamma}}+R_{v} \overline{b^{\beta \gamma}} \overline{v_{\beta \gamma}}\right)+\cdots=\sum_{\beta, \gamma}\left(\overline{a^{\beta \gamma}} R_{u} \overline{u_{\alpha \beta \gamma}}+\overline{b^{\alpha \beta \gamma}} R_{v} \overline{v_{\beta \gamma}}\right)+\cdots=0 .
$$

Учитывая, что

$$
\overline{\partial_{\alpha} \partial_{\beta} R_{\gamma}}=\bar{\partial}_{\alpha} \bar{\partial}_{\beta} \bar{\partial}_{\gamma} R=R_{u} \overline{u_{\alpha \beta \gamma}}+R_{v} \overline{v_{\alpha \beta \gamma}}+\cdots=0,
$$

получаем

$$
\sum_{\beta, \gamma}\left(\overline{a^{\beta \gamma}}-\overline{b^{\beta \gamma}}\right) R_{u} \overline{u_{\alpha \beta \gamma}}+\cdots=0
$$

так что

$$
\overline{a^{\alpha \beta}}=\overline{b^{\alpha \beta}}, \quad 1 \leqslant \alpha, \beta \leqslant D .
$$

\section{4. УТОЧНЕНИЕ ВИДА ДОПУСТИМЫХ ДИФФЕРЕНЦИАЛЬНЫХ СВЯЗЕЙ}

Согласно (2.4) можно положить $\overline{v_{x}}=-\left(R_{u} u_{x}+c_{x}\right) / R_{v}$, считая переменные $u_{x}=$ $\left(u_{\alpha}\right)$ частью координат на поверхности $\mathcal{R}$. Тогда равенство (3.1) примет вид

$$
a^{\alpha \beta}\left(u, u_{x}\right)=b^{\alpha \beta}\left(v, \overline{v_{x}}\right) .
$$

В частности,

$$
a_{u_{\gamma}}^{\alpha \beta}\left(u, u_{x}\right)=\sum_{\delta} b_{v_{\delta}}^{\alpha \beta}\left(v, \overline{v_{x}}\right) \frac{\partial \overline{v_{\delta}}}{\partial u_{\gamma}}=-\frac{R_{u}}{R_{v}} b_{v_{\gamma}}^{\alpha \beta}\left(v, \overline{v_{x}}\right),
$$

или, в симметричном виде,

$$
R_{v} \overline{a_{u_{\gamma}}^{\alpha \beta}}+R_{u} \overline{b_{v_{\gamma}}^{\alpha \beta}}=0 .
$$

Далее,

$$
a_{u}^{\alpha \beta}\left(u, u_{x}\right)=\sum_{\gamma} b_{v_{\gamma}}^{\alpha \beta}\left(v, \overline{v_{x}}\right) \frac{\partial \overline{v_{\gamma}}}{\partial u}=-\sum_{\gamma} b_{v_{\gamma}}^{\alpha \beta}\left(v, \overline{v_{x}}\right)\left[\left(\frac{R_{u}}{R_{v}}\right)_{u} u_{\gamma}+c_{\gamma}\left(\frac{1}{R_{v}}\right)_{u}\right]
$$


так что

$$
a_{u}^{\alpha \beta}\left(u, u_{x}\right)=\sum_{\gamma} \frac{R_{v}}{R_{u}} a^{\alpha \beta}\left(u, u_{x}\right)\left[\left(\frac{R_{u}}{R_{v}}\right)_{u} u_{\gamma}+\left(\frac{1}{R_{v}}\right)_{u} c_{\gamma}\right] .
$$

Взяв производную по $v$ от обеих частей последнего равенства, получим

$$
\begin{aligned}
& {\left[\left(\frac{R_{v}}{R_{u}}\right)\left(\frac{R_{u}}{R_{v}}\right)_{u}\right]_{v}=0, \quad \Rightarrow \quad\left(\ln \frac{R_{u}}{R_{v}}\right)_{u v}=0} \\
& {\left[\left(\frac{R_{v}}{R_{u}}\right)\left(\frac{1}{R_{v}}\right)_{u}\right]_{v}=0, \quad\left(\frac{R_{u v}}{R_{u} R_{v}}\right)_{v}=0}
\end{aligned}
$$

Аналогичным образом выводим

$$
b_{v}^{\alpha \beta}\left(v, v_{x}\right)=\sum_{\gamma} \frac{R_{u}}{R_{v}} b^{\alpha \beta}\left(v, v_{x}\right)\left[\left(\frac{R_{v}}{R_{u}}\right)_{v} v_{\gamma}+\left(\frac{1}{R_{u}}\right)_{v} c_{\gamma}\right]
$$

и

$$
\begin{aligned}
& {\left[\left(\frac{R_{u}}{R_{v}}\right)\left(\frac{R_{v}}{R_{u}}\right)_{v}\right]_{u}=0, \quad \Rightarrow \quad\left(\ln \frac{R_{v}}{R_{u}}\right)_{u v}=0,} \\
& {\left[\left(\frac{R_{u}}{R_{v}}\right)\left(\frac{1}{R_{u}}\right)_{v}\right]_{u}=0, \quad\left(\frac{R_{u v}}{R_{u} R_{v}}\right)_{u}=0 .}
\end{aligned}
$$

Общее решение системы $(4.3),(4.5)$ записывается в виде

$$
R(u, v)=-\frac{1}{c} \ln (M(u)-N(v)), \quad c=\text { const },
$$

где $M, N$ - произвольные функции, причем в силу условия невырожденности (2.1) $M_{u} \neq 0, \quad N_{v} \neq 0$. Без ограничения общности можно положить $c=-1$, так что дифференциальная связь (2.4) принимает вид

$$
R_{\alpha}=\frac{\left(M_{u} u_{\alpha}-N_{v} v_{\alpha}\right)+c_{\alpha}(M-N)}{M-N}=0, \quad 1 \leqslant \alpha \leqslant D,
$$

или, эквивалентно,

$$
R_{\alpha}=\left(\partial_{\alpha}+c_{\alpha}\right) M-\left(\partial_{\alpha}+c_{\alpha}\right) N=0, \quad 1 \leqslant \alpha \leqslant D
$$

\section{5. КООРДИНАТЫ НА ПОВЕРХНОСТИ ДИФФЕРЕНЦИАЛЬНЫХ СВЯЗЕЙ}

Произведем замену переменных в пространстве дифференциальных переменных $u, v, u_{x}, v_{x}, u_{x x}, v_{x x}, \ldots$, а именно, переменные $u, v$ сохраним, переменные первого порядка:

$$
\begin{aligned}
& u_{\alpha}, \quad \rho_{\alpha}=\left(\partial_{\alpha}+c_{\alpha}\right) M, \quad \tau_{\alpha}=\frac{\rho_{\alpha}+\sigma_{\alpha}}{2}, \\
& 1 \leqslant \alpha \leqslant D \\
& v_{\alpha}, \quad \sigma_{\alpha}=\left(\partial_{\alpha}+c_{\alpha}\right) N, \quad \theta_{\alpha}=\frac{\rho_{\alpha}-\sigma_{\alpha}}{2},
\end{aligned}
$$

переменные второго порядка:

$$
\begin{aligned}
& u_{\alpha \beta}, \quad \rho_{\alpha \beta}=\left(\partial_{\alpha} \partial_{\beta}-c_{\alpha} c_{\beta}\right) M, \quad \tau_{\alpha \beta}=\frac{\rho_{\alpha \beta}+\sigma_{\alpha \beta}}{2}, \\
& v_{\alpha \beta}, \quad \sigma_{\alpha \beta}=\left(\partial_{\alpha} \partial_{\beta}-c_{\alpha} c_{\beta}\right) N, \quad \theta_{\alpha \beta}=\frac{\rho_{\alpha \beta}-\sigma_{\alpha \beta}}{2} \\
& 1 \leqslant \alpha, \beta \leqslant D
\end{aligned}
$$


и т.д. (например, $\left.\rho_{\alpha \beta \gamma}=\left(\partial_{\alpha} \partial_{\beta} \partial_{\gamma}+c_{\alpha} c_{\beta} c_{\gamma}\right) M\right)$. В переменных $u, v, \tau_{x}, \theta_{x}, \tau_{x x}, \theta_{x x}, \ldots$ уравнение поверхности дифференциальных связей имеет простой вид:

$$
\mathcal{R}=\left\{\theta_{x}=\theta_{x x}=\cdots=0\right\},
$$

а переменные $u, v, \tau_{x}, \tau_{x x}, \ldots$ суть координаты на $\mathcal{R}$. Сужение функции $F\left(u, v, \tau_{x}, \theta_{x}, \tau_{x x}, \theta_{x x}, \ldots\right)$ на поверхность $\mathcal{R}$ имеет вид

$$
\bar{F}\left(u, v, \tau_{x}, \tau_{x x}, \ldots\right)=F\left(u, v, \tau_{x}, 0, \tau_{x x}, 0, \ldots\right),
$$

а суженные полные дифференцирования -

$$
\bar{\partial}_{\alpha}=\overline{u_{\alpha}} \frac{\partial}{\partial u}+\overline{v_{\alpha}} \frac{\partial}{\partial v}+\sum_{\beta}\left(\tau_{\alpha \beta}+\tau_{\alpha} c_{\beta}\right) \frac{\partial}{\partial \tau_{\beta}}+\sum_{\beta, \gamma}\left(\tau_{\alpha \beta \gamma}-\tau_{\alpha} c_{\beta} c_{\gamma}\right) \frac{\partial}{\partial \tau_{\beta \gamma}}+\cdots
$$

где

$$
\overline{u_{\alpha}}=\frac{1}{M_{u}}\left(\tau_{\alpha}-c_{\alpha} M\right), \quad \overline{v_{\alpha}}=\frac{1}{N_{v}}\left(\tau_{\alpha}-c_{\alpha} N\right), \quad 1 \leqslant \alpha \leqslant D .
$$

В новых координатах

$$
a^{\alpha \beta}=a^{\alpha \beta}\left(u, \rho_{x}\right), \quad b^{\alpha \beta}=b^{\alpha \beta}\left(v, \sigma_{x}\right),
$$

и равенство (3.1) дает

$$
a^{\alpha \beta}\left(u, \tau_{x}\right)=b^{\alpha \beta}\left(v, \tau_{x}\right)=\chi^{\alpha \beta}\left(\tau_{x}\right), \quad 1 \leqslant \alpha, \beta \leqslant D .
$$

Итак, допустимые уравнения (1.2), (1.3) и дифференциальная связь (1.4) имеют вид

$$
\begin{aligned}
u_{t} & =\sum_{\alpha, \beta} \chi^{\alpha \beta}\left(\rho_{x}\right) u_{\alpha \beta}+\phi\left(u, \rho_{x}\right), \\
v_{t} & =\sum_{\alpha, \beta} \chi^{\alpha \beta}\left(\sigma_{x}\right) v_{\alpha \beta}+\psi\left(v, \sigma_{x}\right), \\
R_{\alpha} & =\rho_{\alpha}-\sigma_{\alpha}=\left(\partial_{\alpha}+c_{\alpha}\right) R=0, \quad R=M(u)-N(v), \quad 1 \leqslant \alpha \leqslant D,
\end{aligned}
$$

где $\chi^{\alpha \beta}, \phi, \psi, M, N$ - некоторые функции, $M_{u} \neq 0, N_{v} \neq 0$.

\section{6. СООТВЕТСТВИЯ БЕКЛУНДА. $D>1$}

Приступим к анализу уравнений (5.3)-(5.5). Условие согласования (1.1) имеет вид

$$
\overline{\partial_{t} R_{\alpha}}=\overline{\partial_{t}\left(\partial_{\alpha}+c_{\alpha}\right) R}=\overline{\left(\partial_{\alpha}+c_{\alpha}\right)\left(\partial_{t} R\right)}=\left(\bar{\partial}_{\alpha}+c_{\alpha}\right)\left(\overline{\partial_{t} R}\right)=0
$$

Используя новые переменные, получаем

$$
\begin{aligned}
\overline{\partial_{t} M} & =M_{u} \overline{u_{t}}=\sum_{\alpha, \beta} \chi^{\alpha \beta}\left(\tau_{x}\right) M_{u} \overline{u_{\alpha \beta}}+M_{u} \phi\left(u, \tau_{x}\right)= \\
& =\sum_{\alpha, \beta} \chi^{\alpha \beta}\left(\tau_{x}\right)\left(\tau_{\alpha \beta}-M_{u u} \overline{u_{\alpha} u_{\beta}}+c_{\alpha} c_{\beta} M\right)+M_{u} \phi\left(u, \tau_{x}\right)
\end{aligned}
$$


и аналогичное выражение для $\overline{\partial_{t} N}$, так что

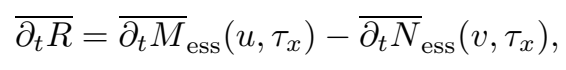

где

$$
\begin{aligned}
& \bar{\partial}_{t} M_{\mathrm{ess}}=\sum_{\alpha, \beta} \chi^{\alpha \beta}\left(\tau_{x}\right)\left(-M_{u u} \overline{u_{\alpha} u_{\beta}}+c_{\alpha} c_{\beta} M\right)+M_{u} \phi\left(u, \tau_{x}\right), \\
& \bar{\partial}_{t} N_{\mathrm{ess}}=\sum_{\alpha, \beta} \chi^{\alpha \beta}\left(\tau_{x}\right)\left(-N_{v v} \overline{v_{\alpha} v_{\beta}}+c_{\alpha} c_{\beta} N\right)+N_{v} \psi\left(v, \tau_{x}\right) .
\end{aligned}
$$

Отсюда

$$
\overline{\partial_{t} R_{\alpha}}=\left(\bar{\partial}_{\alpha}+c_{\alpha}\right)\left(\overline{\partial_{t} R}\right)=\sum_{\beta}\left(\overline{\partial_{t} R}\right)_{\tau_{\beta}} \tau_{\alpha \beta}+\cdots=0,
$$

так что $\left(\overline{\partial_{t} R}\right)_{\tau_{\alpha}}=0,1 \leqslant \alpha \leqslant D$, и, значит,

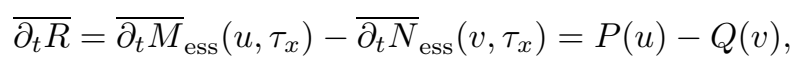

где $P, Q$ - некоторые функции. Следовательно,

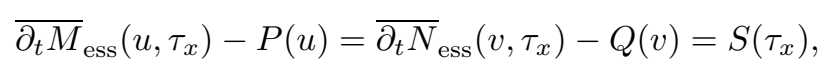

где $S$ - еще одна функция. Таким образом,

$$
\bar{\partial}_{t} M_{\mathrm{ess}}=P(u)+S\left(\tau_{x}\right), \quad \bar{\partial}_{t} N_{\mathrm{ess}}=Q(v)+S\left(\tau_{x}\right) .
$$

Далее, для $1 \leqslant \alpha \leqslant D$ имеем

$$
\overline{\partial_{t} R_{\alpha}}=\left(\bar{\partial}_{\alpha}+c_{\alpha}\right)(P-Q)=\left(P_{u} \overline{u_{\alpha}}+c_{\alpha} P\right)-\left(Q_{v} \overline{v_{\alpha}}+c_{\alpha} Q\right)=0,
$$

откуда в силу $(5.2)$

$$
\frac{P_{u}}{M_{u}}\left(\tau_{\alpha}-c_{\alpha} M\right)+c_{\alpha} P=\frac{Q_{v}}{N_{v}}\left(\tau_{\alpha}-c_{\alpha} N\right)+c_{\alpha} Q,
$$

так что

и, значит,

$$
\frac{P_{u}}{M_{u}}=\frac{Q_{v}}{N_{v}}=c=\text { const }, \quad-c c_{\alpha} M+c_{\alpha} P=-c c_{\alpha} N+c_{\alpha} Q
$$

$$
P(u)=c M(u)+k, \quad Q(v)=c N(v)+k, \quad k=\text { const } .
$$

Без ограничения общности можно положить $k=0$; подставляя полученные выражения в (6.1), получаем

$$
\begin{aligned}
& \overline{\partial t}_{t} M_{\mathrm{ess}}=\sum_{\alpha, \beta} \chi^{\alpha \beta}\left(-M_{u u} \overline{u_{\alpha} u_{\beta}}+c_{\alpha} c_{\beta} M\right)+M_{u} \phi=c M+S, \\
& \bar{\partial}_{t} N_{\mathrm{ess}}=\sum_{\alpha, \beta} \chi^{\alpha \beta}\left(-N_{v v} \overline{v_{\alpha} v_{\beta}}+c_{\alpha} c_{\beta} N\right)+N_{v} \psi=c N+S .
\end{aligned}
$$

Эти равенства завершают описание соответствий Беклунда в изучаемом классе, а именно, доказана следующая теорема. 
Теорема 1. В случае D> 1 уравнения (1.2), (1.3) связаны соответствием Беклунда (1.4) тогда и толъко тогда, когда

$$
\begin{gathered}
a^{\alpha \beta}\left(u, u_{x}\right)=\chi^{\alpha \beta}\left(\rho_{x}\right), \quad b^{\alpha \beta}\left(v, v_{x}\right)=\chi^{\alpha \beta}\left(\sigma_{x}\right), \\
\phi\left(u, u_{x}\right)=\frac{1}{M_{u}}\left(\sum_{\alpha, \beta} \chi^{\alpha \beta}\left(\rho_{x}\right)\left(M_{u u} u_{\alpha} u_{\beta}-c_{\alpha} c_{\beta} M\right)+c M+S\left(\rho_{x}\right)\right), \\
\psi\left(v, v_{x}\right)=\frac{1}{N_{v}}\left(\sum_{\alpha, \beta} \chi^{\alpha \beta}\left(\sigma_{x}\right)\left(N_{v v} v_{\alpha} v_{\beta}-c_{\alpha} c_{\beta} N\right)+c N+S\left(\sigma_{x}\right)\right), \\
R_{\alpha}=\left(\partial_{\alpha}+c_{\alpha}\right) M-\left(\partial_{\alpha}+c_{\alpha}\right) N=0, \quad 1 \leqslant \alpha \leqslant D,
\end{gathered}
$$

где $\chi^{\alpha \beta}\left(\tau_{x}\right), M(u), N(v), S\left(\tau_{x}\right)$ - некоторые функции, $c_{\alpha}, c$-некоторые постолнные, $\rho_{\alpha}=\left(\partial_{\alpha}+c_{\alpha}\right) M, \sigma_{\alpha}=\left(\partial_{\alpha}+c_{\alpha}\right) N, 1 \leqslant \alpha, \beta \leqslant D$, причем $\chi^{\alpha \beta}=\chi^{\beta \alpha}, M_{u} \neq 0$, $N_{v} \neq 0$.

\section{7. СООТВЕТСТВИЯ БЕКЛУНДА. $D=1$}

В этом случае уравнения (1.2)-(1.4) принимают вид (в индексах $\alpha, \beta, \ldots$ больше нет необходимости)

$$
\begin{gathered}
u_{t}=f=a\left(u, u_{x}\right) u_{x x}+\phi\left(u, u_{x}\right), \\
v_{t}=g=b\left(v, v_{x}\right) v_{x x}+\psi\left(v, v_{x}\right), \\
R=R\left(u, u_{x}, v, v_{x}\right)=0,
\end{gathered}
$$

условие (2.2) равенства смешанных производных отсутствует, а условие невырожденности (2.1) имеет вид

$$
R_{u_{x}} \neq 0, \quad R_{v_{x}} \neq 0 .
$$

В частности, уравнение (7.3) разрешимо относительно переменной $v_{x}$ :

$$
v_{x}=r\left(u, u_{x}, v\right), \quad r_{u_{x}} \neq 0,
$$

и условие согласования (1.1) записывается в виде

$$
\begin{aligned}
\overline{\partial_{t}\left(v_{x}-r\right)} & =\bar{b} \overline{v_{x x x}}+\overline{b_{v_{x}}}{\overline{v_{x x}}}^{2}+\cdots-r_{u_{x}}\left(a u_{x x x}+a_{u_{x}} u_{x x}^{2}+\cdots\right)+\cdots= \\
& =r_{u_{x}}(\bar{b}-a) u_{x x x}+\left(\bar{b} r_{u_{x} u_{x}}+\overline{b_{v_{x}}} r_{u_{x}}^{2}-r_{u_{x}} a_{u_{x}}\right) u_{x x}^{2}+\cdots=0,
\end{aligned}
$$

поскольку

$$
\overline{v_{x x}}=r_{u_{x}} u_{x x}+\cdots, \quad \overline{v_{x x x}}=r_{u_{x}} u_{x x x}+r_{u_{x} u_{x}} u_{x x}^{2}+\cdots
$$

Отсюда

$$
\bar{b}-a=0, \quad \bar{b} r_{u_{x} u_{x}}+r_{u_{x}}\left(\overline{b_{v_{x}}} r_{u_{x}}-a_{u_{x}}\right)=0 .
$$

Из первого из этих равенств следует $\overline{b_{v_{x}}} r_{u_{x}}-a_{u_{x}}=0$, так что второе равенство сводится к условию $r_{u_{x} u_{x}}=0$, откуда $r\left(u, u_{x}, v\right)=A(u, v) u_{x}+B(u, v)$ с некоторыми функциями $A$ и $B$. Аналогично $\overline{b_{v_{x}}} r_{u}-a_{u}=0$, откуда

$$
\frac{a_{u}}{a_{u_{x}}}=\frac{r_{u}}{r_{u_{x}}}=\frac{A_{u} u_{x}+B_{u}}{A}=\frac{A_{u}}{A} u_{x}+\frac{B_{u}}{A}
$$


и, значит,

$$
\left(\frac{A_{u}}{A}\right)_{v}=0, \quad\left(\frac{B_{u}}{A}\right)_{v}=0 .
$$

Последовательно решая эти уравнения, находим

$$
A(u, v)=\frac{m(u)}{n(v)}, \quad B(u, v)=\frac{F(u)-G(v)}{n(v)},
$$

где $m \neq 0, n \neq 0, F, G$ - некоторые функции. Таким образом, дифференциальная связь (7.3) записывается как

$$
v_{x}=\frac{m(u)}{n(v)} u_{x}+\frac{F(u)-G(v)}{n(v)}
$$

или, в симметричном виде,

$$
R=\left(\partial_{x} M+F\right)-\left(\partial_{x} N+G\right)=0, \quad M(u)_{u}=m(u), \quad N(v)_{v}=n(v) .
$$

Произведем замену переменных

$$
\begin{array}{llll}
u_{x} & \rho_{x}=\partial_{x} M+F & & \tau_{x}=\frac{\rho_{x}+\sigma_{x}}{2} \\
& \longleftrightarrow & \\
v_{x} & \sigma_{x}=\partial_{x} N+G & \theta_{x}=\frac{\rho_{x}-\sigma_{x}}{2}
\end{array}
$$

затем

$$
\begin{array}{lll}
u_{x x} \quad \rho_{x x}=\partial_{x} \rho_{x} & \\
v_{x x} & \longleftrightarrow \begin{array}{l}
\tau_{x x}=\frac{\rho_{x x}+\sigma_{x x}}{2} \\
\sigma_{x x}=\partial_{x} \sigma_{x}
\end{array} \theta_{x x}=\frac{\rho_{x x}-\sigma_{x x}}{2}
\end{array}
$$

и т.д. (например, $\rho_{x x x}=\partial_{x} \rho_{x x}=\partial_{x}^{2} \rho_{x}$ ). В новых переменных уравнение поверхности дифференциальных связей имеет вид

$$
\mathcal{R}=\left\{\theta_{x}=\theta_{x x}=\cdots=0\right\},
$$

а суженное полное дифференцирование -

$$
\bar{\partial}_{x}=\overline{u_{x}} \frac{\partial}{\partial u}+\overline{v_{x}} \frac{\partial}{\partial v}+\tau_{x x} \frac{\partial}{\partial \tau_{x}}+\tau_{x x x} \frac{\partial}{\partial \tau_{x x}}+\cdots,
$$

где

$$
\overline{u_{x}}=\frac{1}{M_{u}}\left(\tau_{x}-F\right), \quad \overline{v_{x}}=\frac{1}{N_{v}}\left(\tau_{x}-G\right) .
$$

Далее, $a=a\left(u, \rho_{x}\right), b=b\left(v, \sigma_{x}\right)$, и первое из равенств $(7.5)$ дает

$$
a\left(u, \tau_{x}\right)=b\left(v, \tau_{x}\right)=\chi\left(\tau_{x}\right) .
$$

Итак, допустимые уравнения (7.1), (7.2) и дифференциальная связь (7.3) имеют вид

$$
u_{t}=\chi\left(\rho_{x}\right) u_{x x}+\phi\left(u, \rho_{x}\right) \text {, }
$$




$$
\begin{aligned}
v_{t} & =\chi\left(\sigma_{x}\right) v_{x x}+\psi\left(v, \sigma_{x}\right), \\
R & =\rho_{x}-\sigma_{x}=0,
\end{aligned}
$$

где $\rho_{x}=\partial_{x} M(u)+F(u), \sigma_{x}=\partial_{x} N(v)+G(v)$, а $\chi, \phi, \psi, M, F, N, G$ - некоторые функции, $M_{u} \neq 0, N_{v} \neq 0$.

Условие согласования (1.1) имеет вид

$$
\begin{aligned}
\overline{\partial_{t} R} & =\left(\overline{\partial_{t} \partial_{x} M}+\overline{\partial_{t} F}\right)-\left(\overline{\partial_{t} \partial_{x} N}+\overline{\partial_{t} G}\right)= \\
& =\left(\bar{\partial}_{x}+\mu\right) \overline{\partial_{t} M}-\left(\bar{\partial}_{x}+\nu\right) \overline{\partial_{t} N}=0, \quad \mu=\frac{F_{u}}{M_{u}}, \quad \nu=\frac{G_{v}}{N_{v}}
\end{aligned}
$$

Здесь

$$
\begin{aligned}
\overline{\partial_{t} M}=\chi\left(\tau_{x}\right) \tau_{x x}+K, \quad K\left(u, \tau_{x}\right)=-\chi\left(\tau_{x}\right)\left(M_{u u}{\overline{u_{x}}}^{2}+F_{u} \overline{u_{x}}\right)+M_{u} \bar{\phi}, \\
\overline{\partial_{t} N}=\chi\left(\tau_{x}\right) \tau_{x x}+L, \quad L\left(v, \tau_{x}\right)=-\chi\left(\tau_{x}\right)\left(N_{v v}{\overline{v_{x}}}^{2}+G_{v}{\overline{v_{x}}}\right)+N_{v} \bar{\psi},
\end{aligned}
$$

так что

$$
\begin{aligned}
\overline{\partial_{t} R} & =\left[\bar{\partial}_{x} K+\mu\left(\chi \tau_{x x}+K\right)\right]-\left[\bar{\partial}_{x} L+\nu\left(\chi \tau_{x x}+L\right)\right]= \\
& =[(K+\mu X)-(L+\nu X)]_{\tau_{x}} \tau_{x x}+\left[\left(K_{u} \overline{u_{x}}+\mu K\right)-\left(L_{v} \overline{v_{x}}+\nu L\right)\right]=0,
\end{aligned}
$$

где

$$
\chi\left(\tau_{x}\right)=X_{\tau_{x}}\left(\tau_{x}\right), \quad X(0)=0
$$

Итак,

$$
\begin{aligned}
{[(K+\mu X)-(L+\nu X)]_{\tau_{x}} } & =0, \\
\left(K_{u} \overline{u_{x}}+\mu K\right)-\left(L_{v} \overline{v_{x}}+\nu L\right) & =0,
\end{aligned}
$$

откуда

$$
\begin{aligned}
K+\mu X & =P(u)+S\left(\tau_{x}\right), & K_{u} \overline{u_{x}}+\mu K & =T\left(\tau_{x}\right), \\
L+\nu X & =Q(v)+S\left(\tau_{x}\right), & L_{v} \overline{v_{x}}+\nu L & =T\left(\tau_{x}\right),
\end{aligned}
$$

где $P, Q, S, T$ - некоторые функции, причем без ограничения общности считаем $S(0)=0$. Полный анализ уравнений $(7.12)$, (7.13) требует рассмотрения ряда частных случаев и является достаточно громоздким. Поскольку наша цель - изучение преобразований Беклунда эволюционных систем в многомерном пространстве $(D>1)$, мы ограничимся здесь случаем $\mu=$ const $\neq 0, \quad \nu=$ const $\neq 0$. Тогда без ограничения общности можно считать $F=\mu M, G=\nu N$ (заметим, что функции $M(u), N(v)$ определены с точностью до постоянной), и правые уравнения системы (7.12), (7.13) имеют общие решения

$$
K\left(u, \tau_{x}\right)=\frac{T\left(\tau_{x}\right)}{\mu}+\kappa\left(\tau_{x}\right)\left(\tau_{x}-F(u)\right), \quad L\left(v, \tau_{x}\right)=\frac{T\left(\tau_{x}\right)}{\nu}+\lambda\left(\tau_{x}\right)\left(\tau_{x}-G(v)\right),
$$

где $\kappa, \lambda$ - некоторые функции. Далее, левые уравнения системы (7.12), (7.13) дают $\kappa=$ const,$\quad \lambda=$ const $и$

$$
P(u)=-\kappa F(u)+\frac{T(0)}{\mu}, \quad S\left(\tau_{x}\right)=\frac{T\left(\tau_{x}\right)-T(0)}{\mu}+\kappa \tau_{x}+\mu X\left(\tau_{x}\right),
$$




$$
Q(v)=-\lambda G(v)+\frac{T(0)}{\nu}, \quad S\left(\tau_{x}\right)=\frac{T\left(\tau_{x}\right)-T(0)}{\nu}+\lambda \tau_{x}+\nu X\left(\tau_{x}\right)
$$

(напомним, что $S(0)=0$ ). Эти рассуждения приводят к следующему достаточному условию существования соответствия Беклунда.

TEOPEMA 2. Пусть $D=1 u$

$$
\begin{aligned}
& \phi\left(u, u_{x}\right)=\frac{1}{M_{u}}\left(\chi\left(\rho_{x}\right)\left(M_{u u} u_{x}^{2}+\mu M_{u} u_{x}\right)+\frac{T\left(\rho_{x}\right)}{\mu}+\kappa M_{u} u_{x}\right) \\
& \psi\left(v, v_{x}\right)=\frac{1}{N_{v}}\left(\chi\left(\sigma_{x}\right)\left(N_{v v} v_{x}^{2}+\nu N_{v} v_{x}\right)+\frac{T\left(\sigma_{x}\right)}{\nu}+\lambda N_{v} v_{x}\right)
\end{aligned}
$$

где $\rho_{x}=\left(\partial_{x}+\mu\right) M, \sigma_{x}=\left(\partial_{x}+\nu\right) N, \chi\left(\tau_{x}\right)=X_{\tau_{x}}\left(\tau_{x}\right), M(u), N(v)-$ произвольнье функиии, а функции $T\left(\tau_{x}\right), X\left(\tau_{x}\right)$ и постоянные $\mu, \nu, \kappa, \lambda$ удовлетворяют условию согласования

$$
\left(\frac{1}{\mu}-\frac{1}{\nu}\right) T\left(\tau_{x}\right)+(\kappa-\lambda) \tau_{x}+(\mu-\nu) X\left(\tau_{x}\right)=0 .
$$

Тогда уравнения (7.7), (7.8) связаны соответствием Беклунда (7.9).

\section{8. ЗАКЛЮЧЕНИЕ}

Главный итог предлагаемой работы - демонстрация возможности эффективного алгебро-геометрического анализа уравнений в частных производных с произвольным числом независимых переменных. Конкретным результатом является полное описание важного класса уравнений многомерной эволюции, допускающих соответствия Беклунда заданного вида. В частности, оказалось, что изученный класс достаточно широк, хотя в одномерном случае он богаче благодаря отсутствию условия равенства смешанных производных.

Благодарности. Работа выполнена при поддержке РФФИ (грант № 04-01-00769) и Программы поддержки ведущих научных школ (грант № НШ-1542.2003.1).

\section{Список литературы}

[1] В. В. Жаринов, Матем. сб., 136(178):2 (1988), 274-291.

[2] V.V. Zharinov, Lecture Notes on Geometrical Aspects of Partial Differential Equations, (Ser. Sov. Fas Eur. Math., Vol. 9), World Scientific, Singapore, 1992.

[3] I. S. Krasil'shchik, A. M. Vinogradov, Acta. Appl. Math., 15 (1989), 161-209.

[4] Л.В. Овсянников, Групповой анализ дифференциальных уравнений, Наука, М., 1978; Н.Х. Ибрагимов, Группь преобразований в математической физике, Наука, М., 1983; П. Олвер, Приложения групп Ли к дифференциальным уравнениям, Мир, М., 1989; А.М. Виноградов, И.С. Красильщик (ред.), Симметрии и законы сохранения уравнений математической физики, Факториал, М., 1997; В. J. Cantwell, Introduction to Symmetry Analysis, Camb. Univ. Press, Cambridge, 2002. 